\title{
Visual Analysis of the Daily QA Results of Photon and Electron Beams of a Trilogy Linac over a Five-Year Period
}

\author{
Maria F. Chan1, Qiongge Li², Xiaoli Tang1, Xiang Li1, Jingdong Li1, Grace Tang1, \\ Margie A. Hunt ${ }^{1}$, Joseph 0. Deasy ${ }^{1}$ \\ ${ }^{1}$ Department of Medical Physics, Memorial Sloan Kettering Cancer Center, New York, USA \\ ${ }^{2}$ Department of Physics, The City University of New York, New York, USA \\ Email: chanm@mskcc.org
}

Received 15 August 2015; accepted 6 November 2015; published 9 November 2015

Copyright (C) 2015 by authors and Scientific Research Publishing Inc.

This work is licensed under the Creative Commons Attribution International License (CC BY). http://creativecommons.org/licenses/by/4.0/

(c) (i) Open Access

\begin{abstract}
Data visualization technique was applied to analyze the daily QA results of photon and electron beams. Special attention was paid to any trend the beams might display. A Varian Trilogy Linac equipped with dual photon energies and five electron energies was commissioned in early 2010. Daily Linac QA tests including the output constancy, beam flatness and symmetry (radial and transverse directions) were performed with an ionization chamber array device (QA Beam Checker Plus, Standard Imaging). The data of five years were collected and analyzed. For each energy, the measured data were exported and processed for visual trending using an in-house Matlab program. These daily data were cross-correlated with the monthly QA and annual QA results, as well as the preventive maintenance records. Majority of the output were within $1 \%$ of variation, with a consistent positive/upward drift for all seven energies ( $+\mathbf{+ 0 . 2 5 \%}$ per month). The baseline of daily device is reset annually right after the TG-51 calibration. This results in a sudden drop of the output. On the other hand, the large amount of data using the same baseline exhibits a sinusoidal behavior (period $=12$ months; amplitude $=0.8 \%, 0.5 \%$ for photons, electrons, respectively) on symmetry and flatness when normalization of baselines is accounted for. The well known phenomenon of new Linac output drift was clearly displayed. This output drift was a result of the air leakage of the over-pressurized sealed monitor chambers for the specific vendor. Data visualization is a new trend in the era of big data in radiation oncology research. It allows the data to be displayed visually and therefore more intuitive. Based on the visual display from the past, the physicist might predict the trend of the Linac and take actions proactively. It also makes comparisons, alerts failures, and potentially identifies causalities.
\end{abstract}

\section{Keywords}

Linac QA, Data Visualization, Daily Output, Radiotherapy 


\section{Introduction}

Pre-treatment daily dosimetry monitoring including X-ray and electron output constancy together with the functional safety checks is recommended for all medical accelerators [1]-[3]. The daily tests include parameters that can affect dose to the patient, such as dosimetric output constancy, geometric accuracy of lasers, optical distance indicator, and field size, as well as other safety tests. These daily tests are typically carried out by the morning warm-up radiation therapist using a cross-calibrated dosimetry system. Commercial flat-panel multidetector arrays with appropriate build up material may be also used for daily quality assurance (QA). The absolute dose output and other dosimetry tests are also performed in the monthly QA test with higher level equipment at tighter tolerance criteria. This provides confidence in the daily device and will identify trends that may otherwise go undetected over the course of a long period of time.

In addition to keeping parameters within specification, it is important for a physicist to monitor trends in the machine performance. A well-designed trending tool may help to predict future problems and allow proper planning of action level [4]. Large longitudinal data visualization analysis differs from traditional statistical analysis because it is dynamic due to its data capacity and content, and more advanced processing methods have made it possible to analyze the dynamic visualization [5]. The objective of this study is to utilize data visualization to trend the behavior of photon and electron beams of a new linear accelerator (Linac) in our radiotherapy center. This work devotes to discovering the common behaviors of the Linac output, so that physicists can be more confident in predicting machine's future behavior and taking action in a planned way before the tolerance level is reached. Preventive adjustment is more efficient than post-event action (adjusting after the tolerance level is reached); on the other hand, this intuitive adjustment grounding on everyday observations can be more economical than the more frequent prescribed preventive maintenance.

\section{Materials and Methods}

A Trilogy Linac (Varian Medical Systems, Palo Alto, CA) equipped with dual photon energies (6 and 15 MV) and five electron energies $(6,9,12,16$, and $20 \mathrm{MeV}$ ) was commissioned in early 2010. Daily Linac QA tests including the output constancy, flatness, and beam symmetry (for both radial and transverse directions) were measured with an ionization chamber array device, QABeam Checker Plus (QABC+) (Standard Imaging Inc., Wisconsin, US), for the past five years (3/16/2010-3/02/2015). There are 8 vented ion-chambers in the device: 1 center detector, 4 quadrant detectors ( $7.5 \mathrm{~cm}$ from center), and 3 energy identification chambers. Figure 1(a) and Figure 1(b) show the setup of QABC+ on a Linac couch for QA tests and the device flips on different side for photon/electron measurement. This task is performed by a radiation therapist on a daily basis to ensure the Linac output within tolerances in compliance with the AAPM TG142 recommendation. All data are stored in the database of the QABC+ software. Figure 2 demonstrates a set of typical unfiltered raw data extracted from the daily device. Figure 3(a) shows the $6 \mathrm{MV}$ raw data extracted from QABC+; and Figure 3(b) plots the $6 \mathrm{MV}$ output and axial symmetry (CONSTANCY vs. AXSYM) along the timeline over 5-year period. CONSTANCY is in percentage of the output variation to the baseline; AXSYM is beam symmetry along the axial direction; TRSYM is symmetry along the transverse direction; and FLATNESS is uniformity over the radiation field.

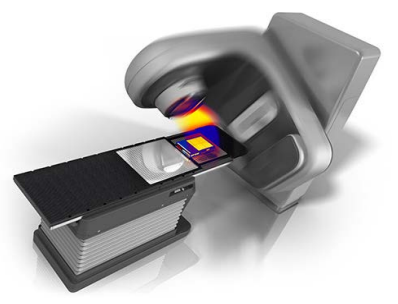

(a)

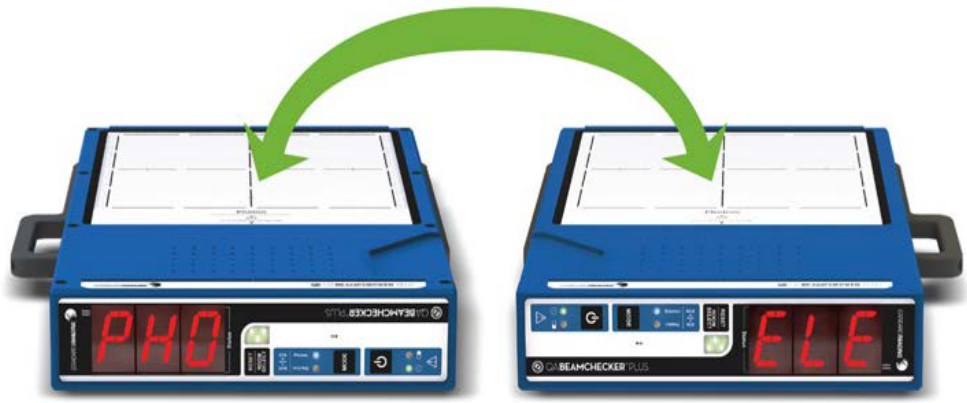

(b)

Figure 1. (a) QABC+ on a Linac couch to measure daily outputs for all 7 energies before treating cancer patients; (b) $\mathrm{QABC}+$ device: flips between photons and electrons measurement. 


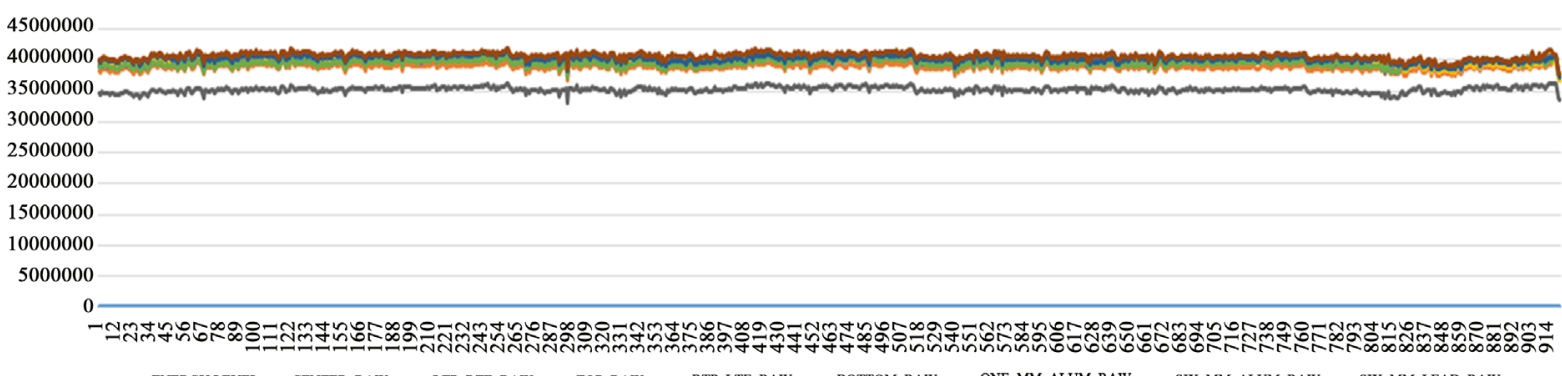

Figure 2. Unfiltered raw data extracted from QABC+ for 5-year period.

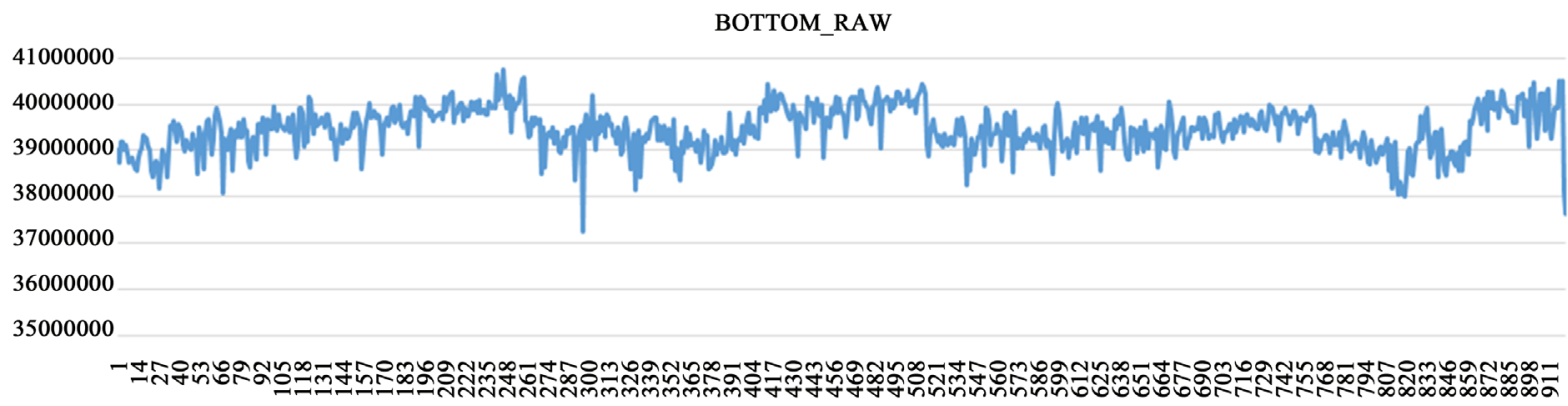

(a)

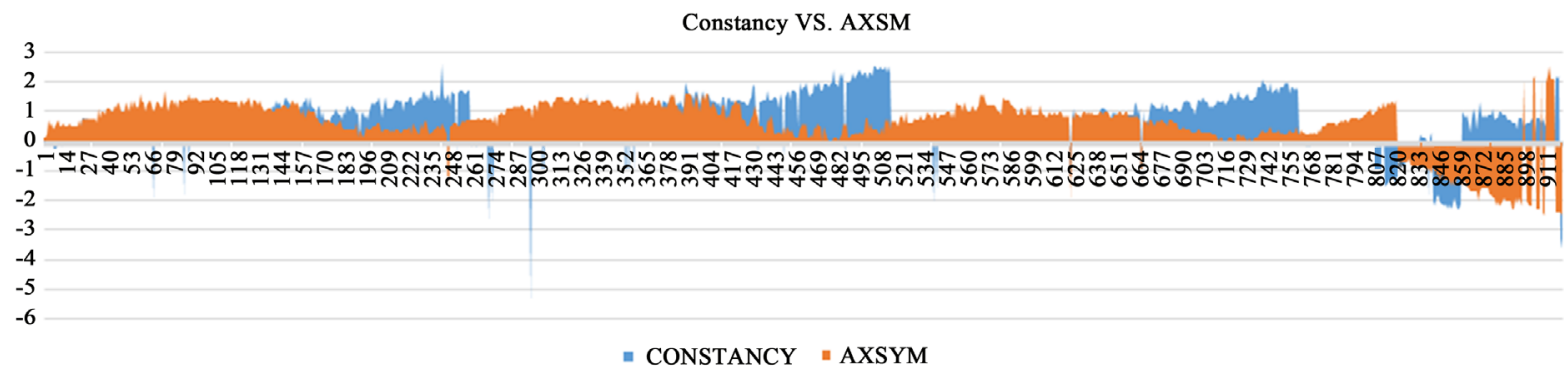

(b)

Figure 3. (a) $6 \mathrm{MV}$ raw data extracted from QABC+; (b) combined $6 \mathrm{MV}$ output constancy and axial symmetry along the timeline on the $\mathrm{X}$-axis. The $\mathrm{Y}$-axis represent of the percentage difference from baseline.

After the measurement data were exported for each of the seven energies, they were then processed for visual trending using an in-house program developed with Matlab (v8.1.0.604). Output constancy data were plotted for all energies. For both photon energies, the symmetry plots were fitted with a third-order sinusoidal function and the flatness plot was fitted with sum of sine with a $4^{\text {th }}$ order function (the curve fitting tool provided by Matlab). These daily measurement data were cross-correlated with monthly QA and annual QA data, as well as the preventive maintenance records.

In addition, a multiple regression analysis was performed by creating the scatter plot matrix that provides a quick detection of the correlation between variables. The scatter plot matrix for all seven energies were created by the commercial statistical software Minitab 17 (Windows). Each variable pair is the same-day measurements. Linear regression model was fitted in each of these plots. The correlation coefficients were calculated by using Matlab statistical function corrplot().

\section{Results}

The output of the Linac is mostly within $1 \%$ of variation, with a consistent positive drift for all seven energies ( $\sim+0.25 \%$ per month). The output is adjusted and corrected every year in September, when annual TG-51 cali- 
bration is performed. This results in the sudden drops of output back to the baseline in every September in the data trend. Figure 4 and Figure 5 are the constancy plots for photons and electrons over the 5-year period, respectively.

The large amount of data using the same baseline exhibits a sinusoidal behavior (period = 12 months; amplitude $=0.8 \%, 0.5 \%$ for photons, electrons, respectively) on symmetry and flatness when normalization of baselines is accounted for. Figures 6-11 are the AXSYM, TRSYM, and FLATNESS plots for photons and electrons over the 5-year period, respectively. The photon plots were fitted with a sinusoidal function (small windows). We did not show the fitting function for the electron plots for better clarity.

A scatter plot matrix helps knowing one dosimetric parameter's behavior, which could assist predicting others. Figures 12(a)-(e) show the scatter plot matrix for all seven energies. The scatter plot matrix for photons (both $6 \mathrm{MV}$ and $15 \mathrm{MV}$ ) shows relatively strong negative correlation between AXSYM and FLATNESS (correlation coefficient (CC): -0.67 and -0.71 ), negative correlations between AXSYM and TRSYM (CC: -0.51 and -0.76 ), and positive correlations between TRSYM and FLATNESS (CC: 0.67 and 0.62). However, for different elec-

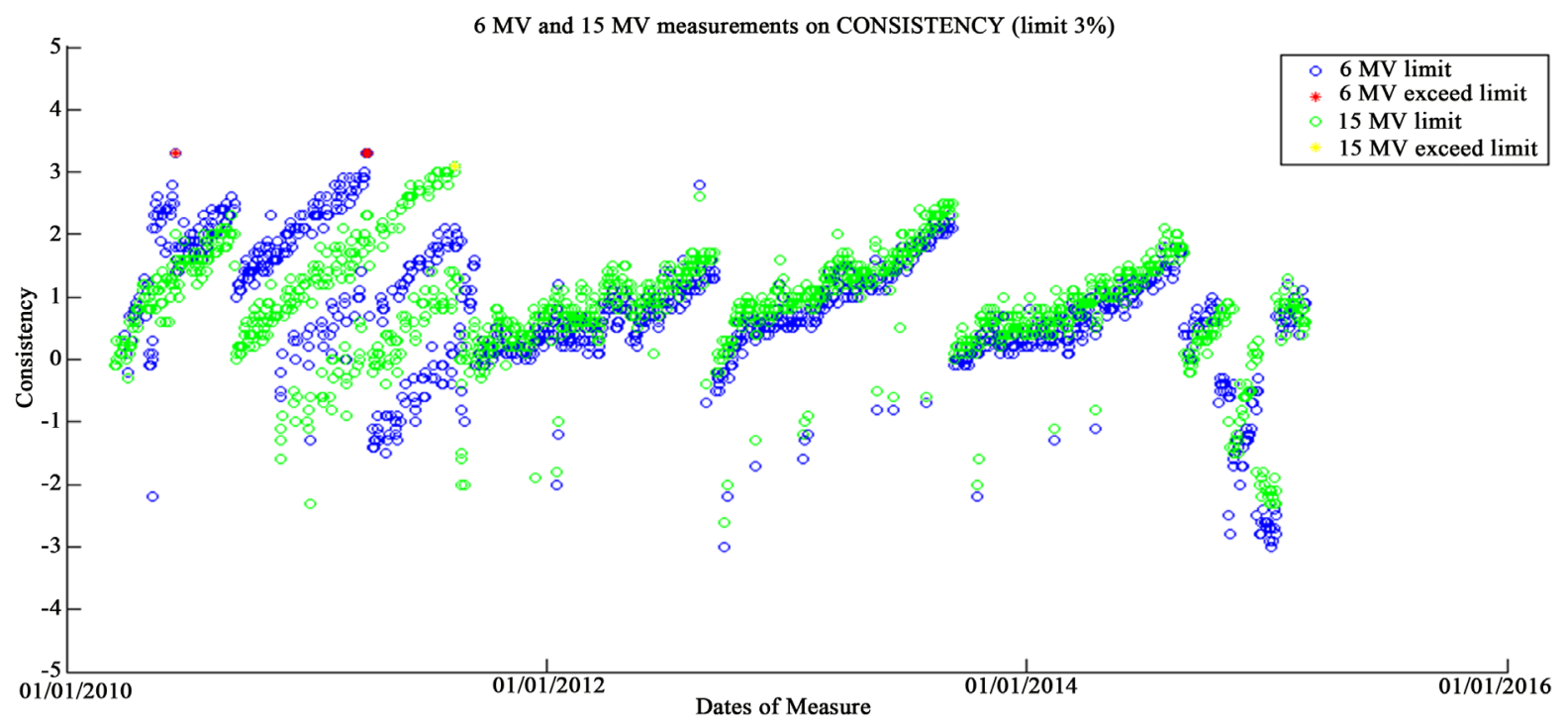

Figure 4. Constancy of two photon energies in 5-year timeline.

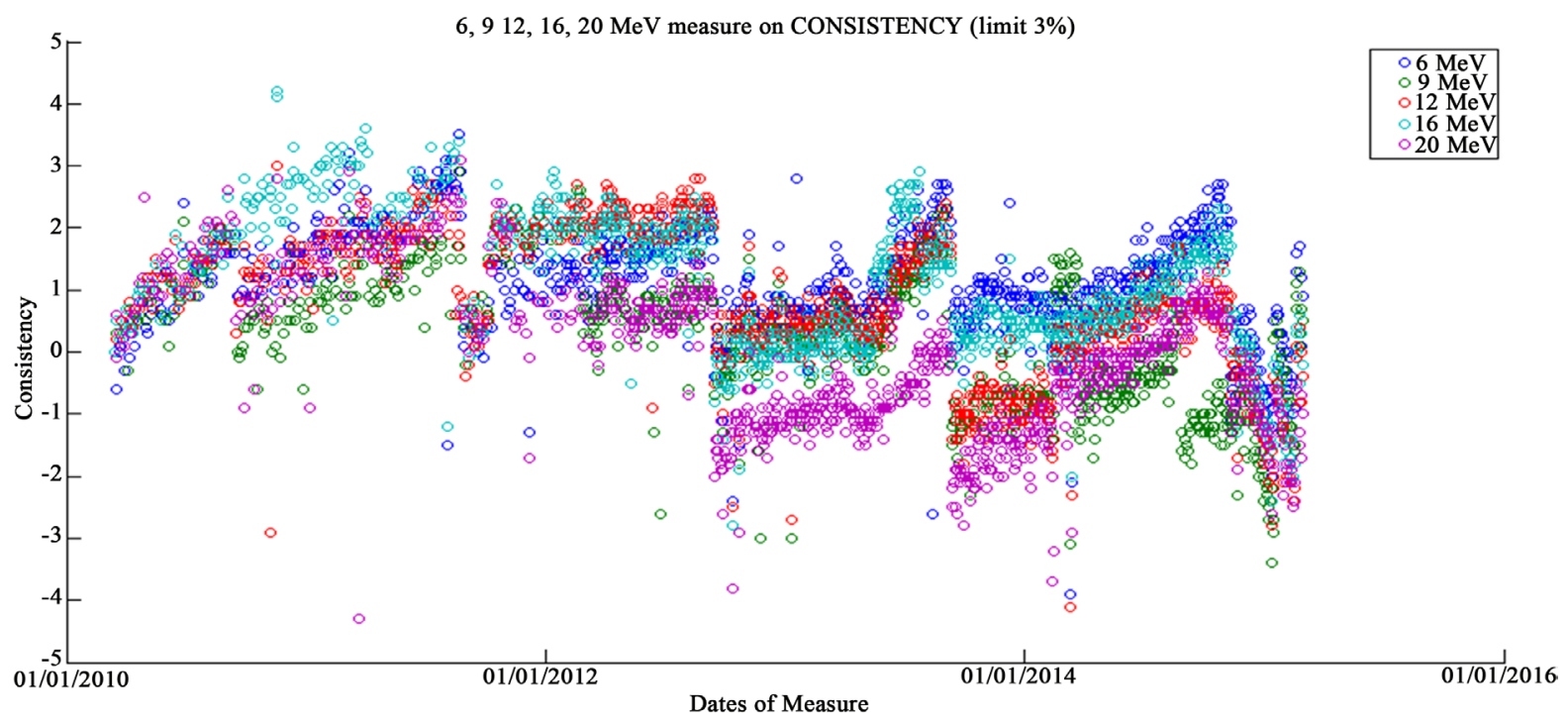

Figure 5. Constancy of five electron energies in 5-year timeline. 


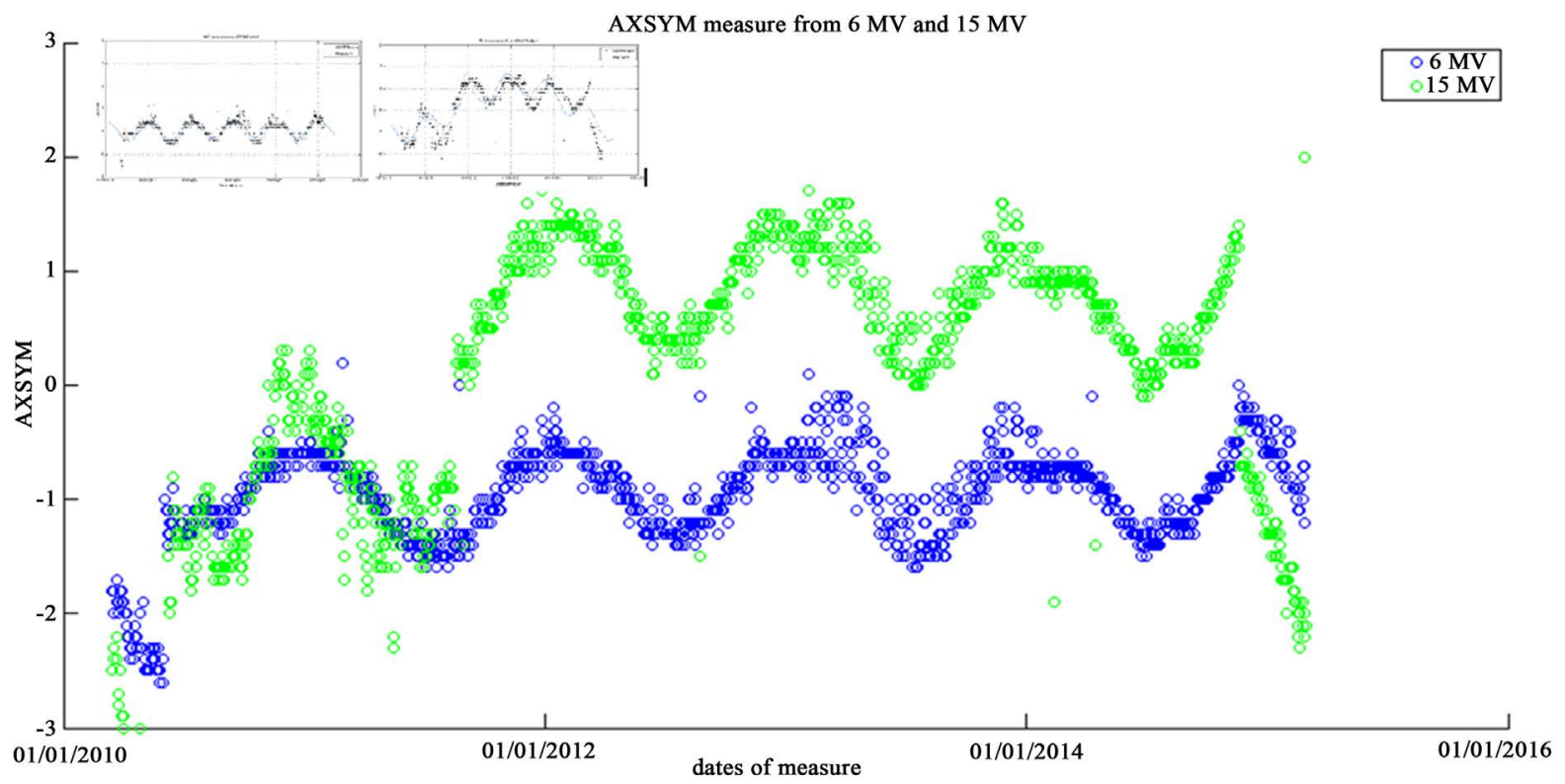

Figure 6. Filtered photon axial symmetry data with curve fitting functions in the 2 separate plots.

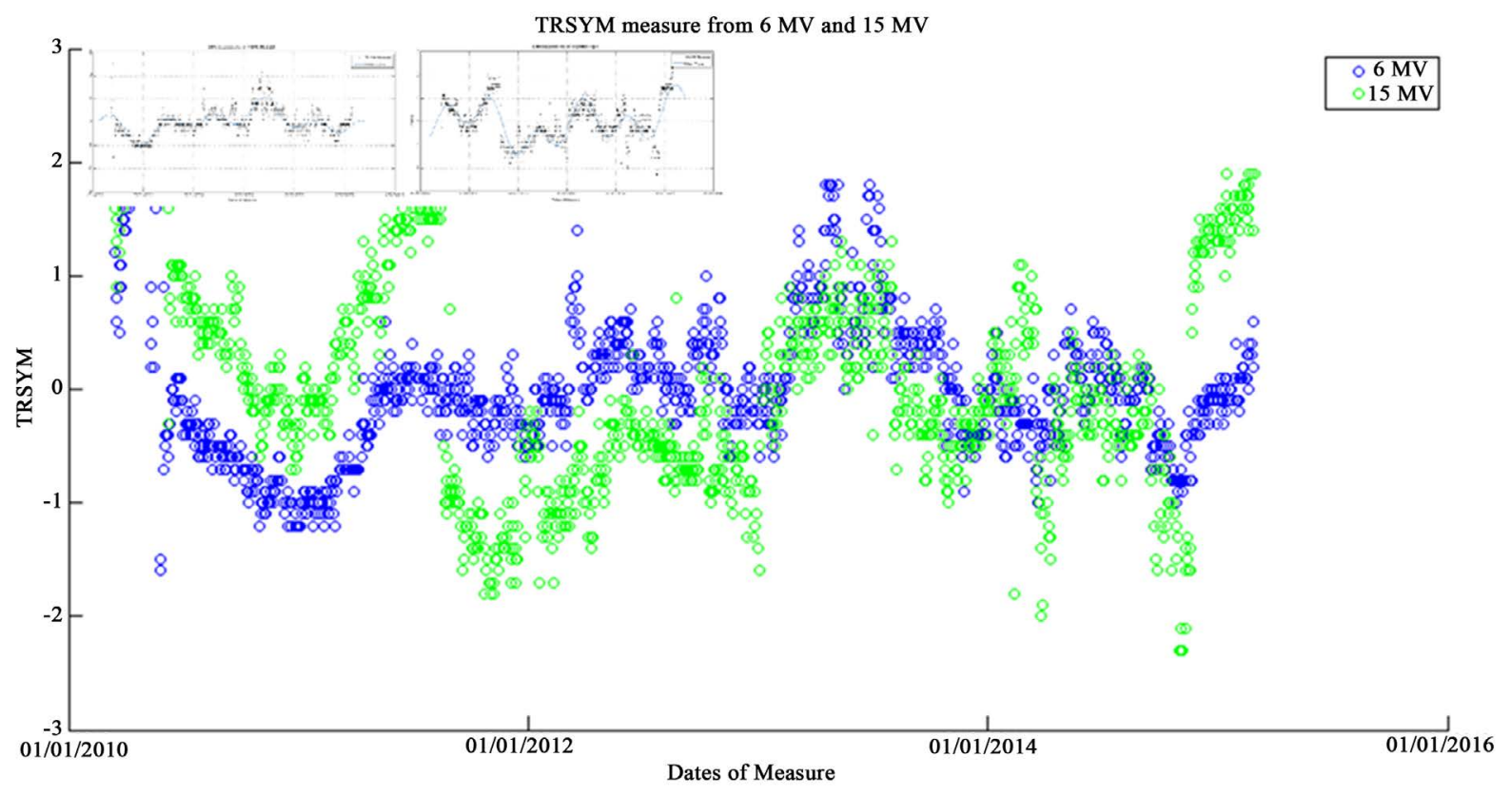

Figure 7. Filtered photon transverse symmetry data with curve fitting functions in the 2 separate plots.

tron energies, this correlation relationship is not consistent: AXSYM and TRSYM show strong positive correlation (CC: 0.78 ) in $6 \mathrm{MeV}$ electron plot, while negative correlation (CC: -0.52) in $16 \mathrm{MeV}$ electron plot, and no correlation in other energies (-0.25 - 0.29). AXSYM and FLATNESS show positive correlation (CC: 0.62) only in $6 \mathrm{MeV}$ electron plot and almost no apparent correlation in other energies (CC: $-0.4-0.26$ ). TRSYM and FLATNESS show positive correlation in $6 \mathrm{MeV}$ energy electron plot (CC: 0.52 ) but negative correlations in 9, 12, and $16 \mathrm{MeV}$ electrons (CC: $-0.52,-0.57,-0.59$ ), and no correlation (CC: -0.12 ) in $20 \mathrm{MeV}$ plot. Over all seven energies, CONSTANCY is not correlated to any of the other three variables, while there are correlations among the symmetry and flatness for both photon energies. For electrons, only 6MeV shows correlations between symmetry and flatness, similar to photons. The least correlation among variables is $20 \mathrm{MeV}$. The order 


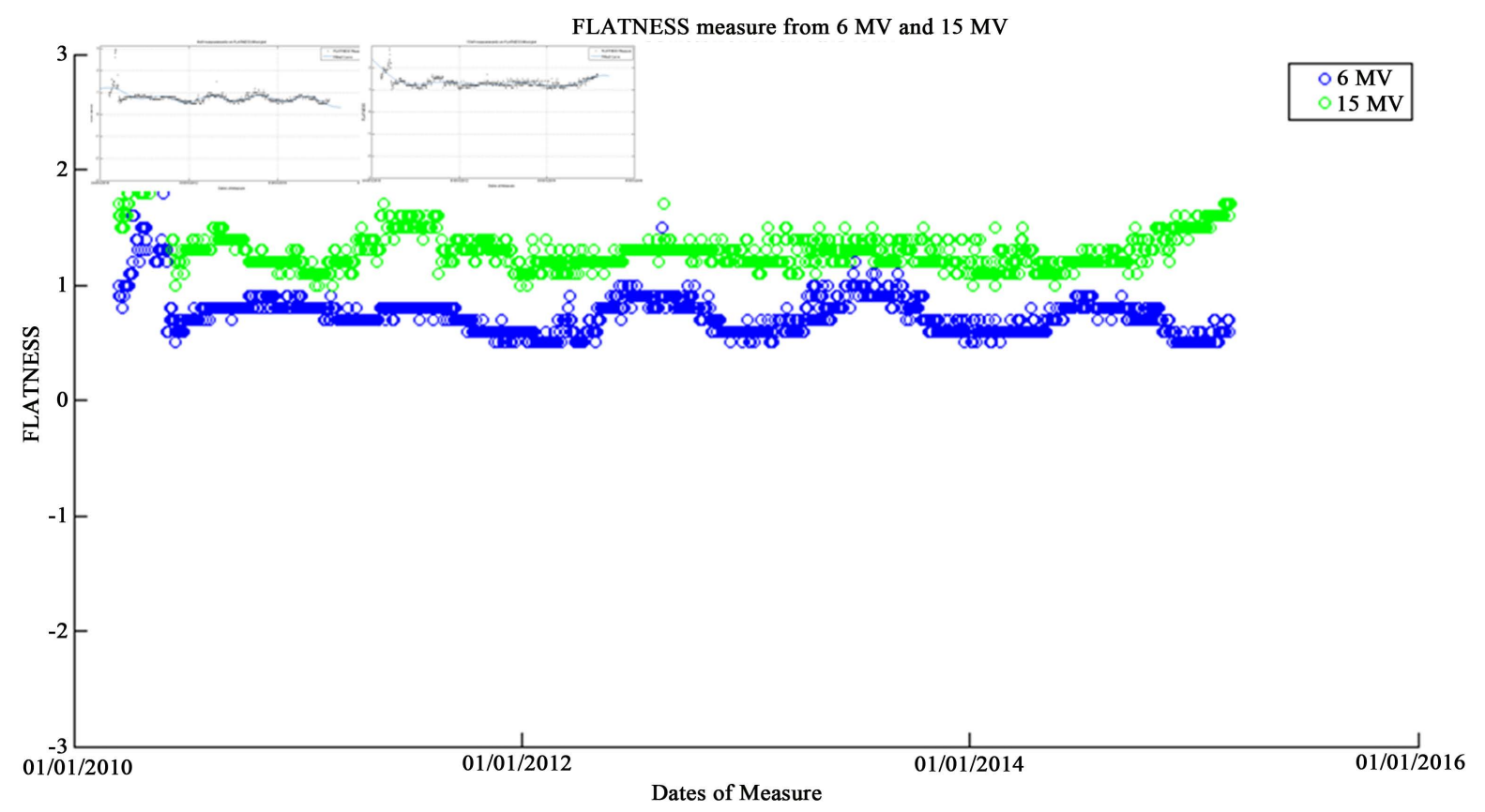

Figure 8. Filtered photon flatness data with curve fitting functions in the 2 separate plots.

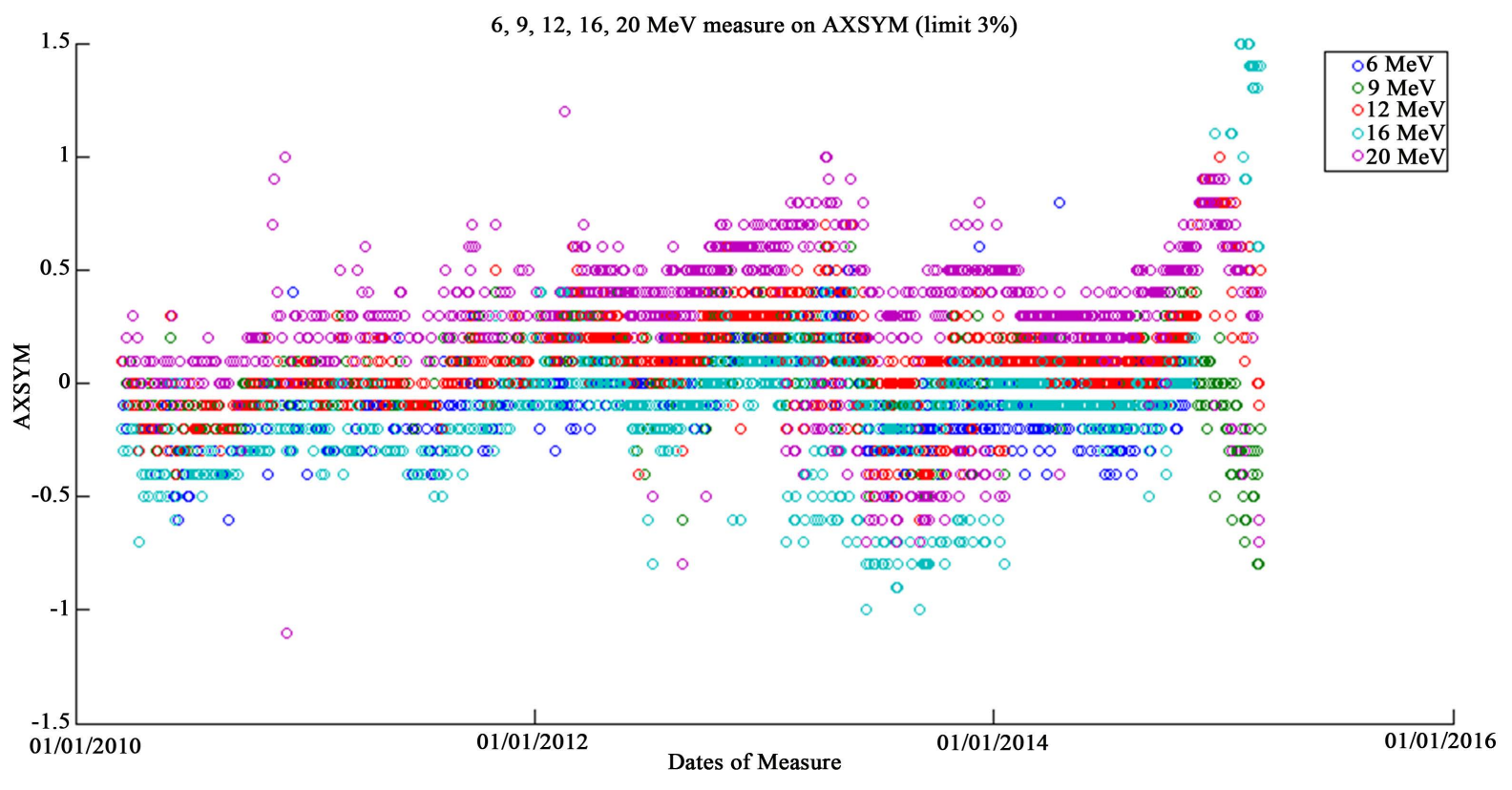

Figure 9. Axial symmetry for five electron energies.

from strong to week correlations in electrons is $6 \mathrm{MeV}, 16 \mathrm{MeV}, 9 \mathrm{MeV}, 12 \mathrm{MeV}$, and $20 \mathrm{MeV}$, respectively. The knowledge gained from this study would help the physicist better understand the different behavior or character for each beam.

\section{Discussion}

The output of the Linac is mostly within $1 \%$ of variation, with a consistent positive drift for all seven energies ( $+0.25 \%$ per month), which is closely consistent with Grattan and Hounsell's report [6] on "average monthly increase of $0.26 \%+/-0.009 \%$ over the course of the first 4 years of use”. However, in their study, they reported 


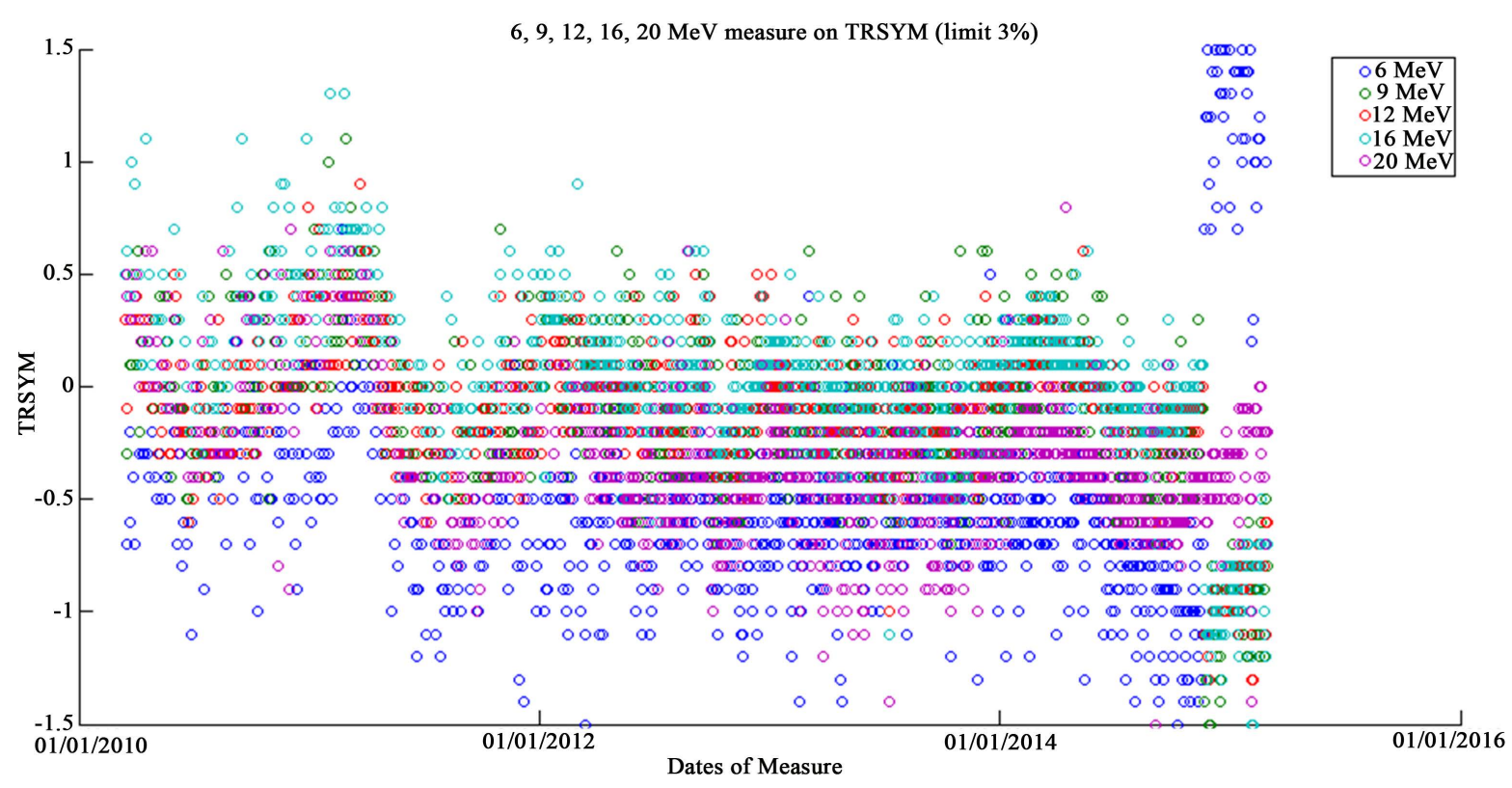

Figure 10. Transverse symmetry for five electron energies.

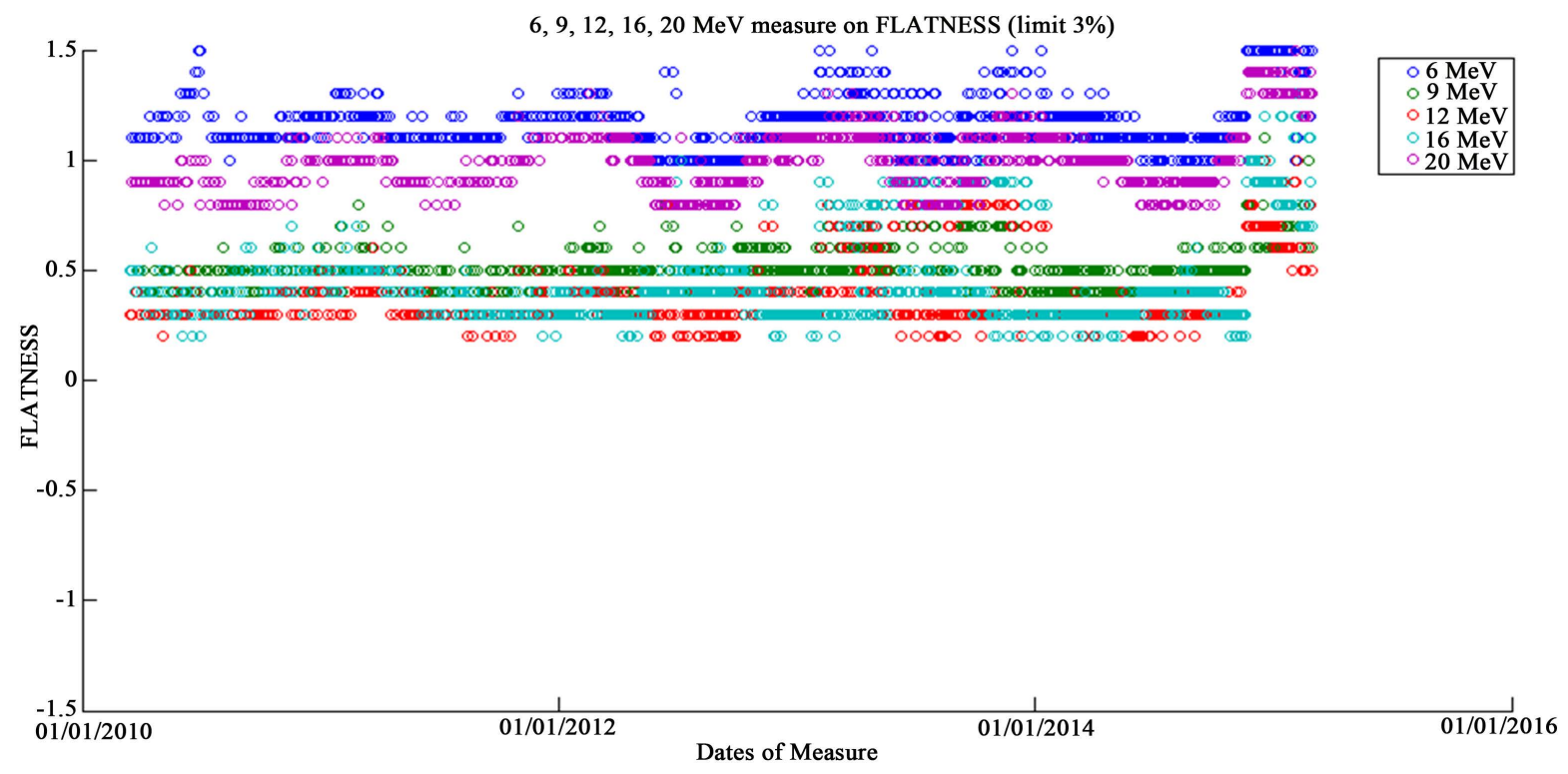

Figure 11. Flatness (data combined both axial and transverse) for five electron energies.

that the output increase then reduces to $-0.03 \%+/-0.002 \%$ for the following three years. For this aspect, we will continue to monitor the trend and see if this would be in the same direction as theirs. The phenomenon of output drift for new Linacs is due to air leakage of the over-pressurized sealed monitor chambers made by the specific vendor [6]. Furthermore, our data is very consistent with the seasonal fluctuation (quasi-periodic behavior) found in the previous studies [6] and [7]. Particularly, the sinusoidal behavior is in agreement with the observations in seasonal variation described by other researchers [6]-[8]. This is probably due to the incoming power to the circuit boards, which can be affected by the environmental factors year-round, and the varying output that could also cause more and less scatter from the electronics and structures surrounding the chambers in the $\mathrm{QABC}+$. The environmental factors include the fixed timing of the startup of the air conditioning and heating in our building.

Grattan and Hounsell [6] reported their analysis of Varian linear accelerators output trends up to 7 years. The 


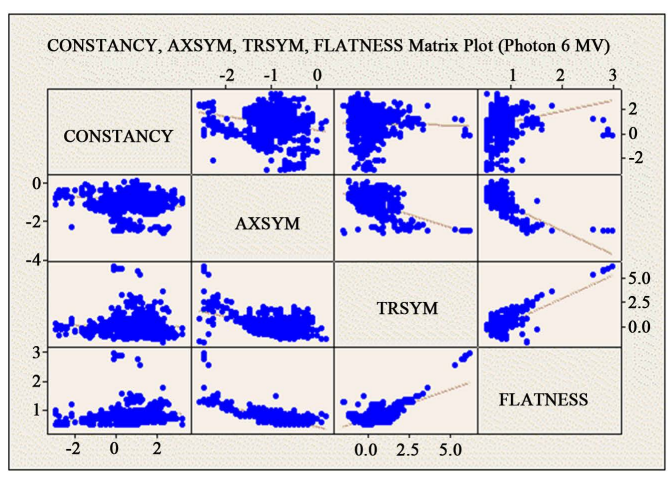

(a)

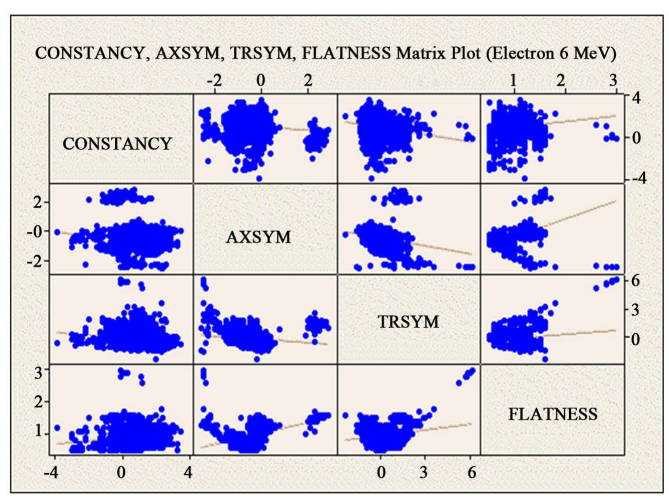

(c)

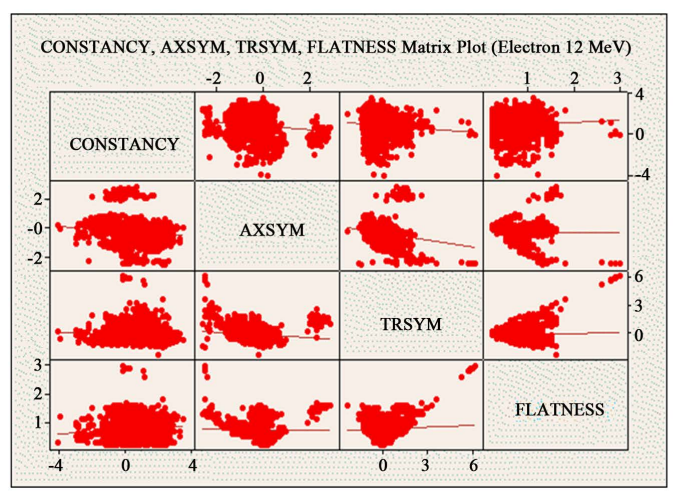

(e)

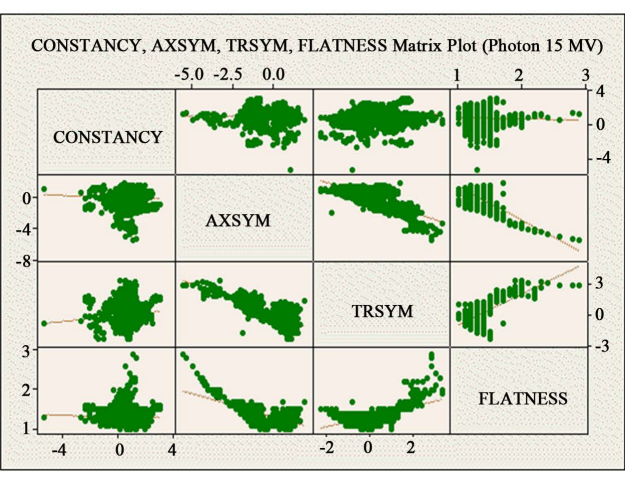

(b)

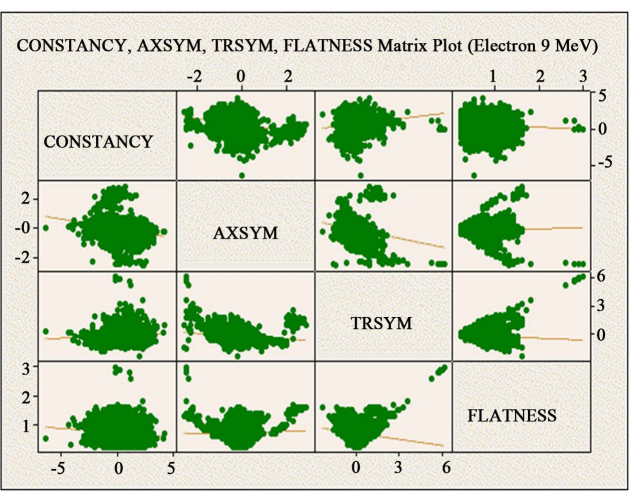

(d)

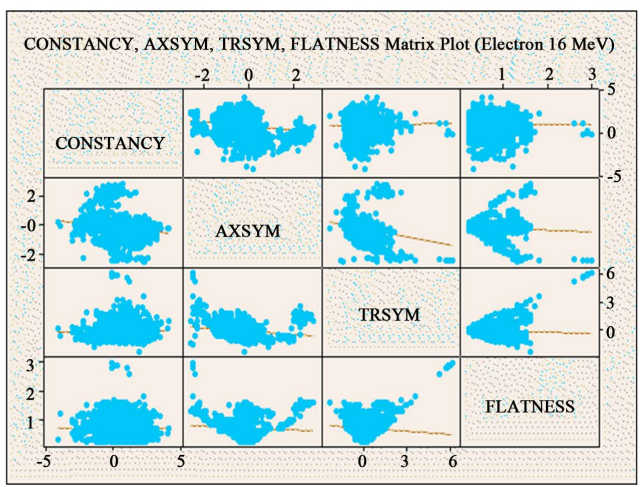

(f)

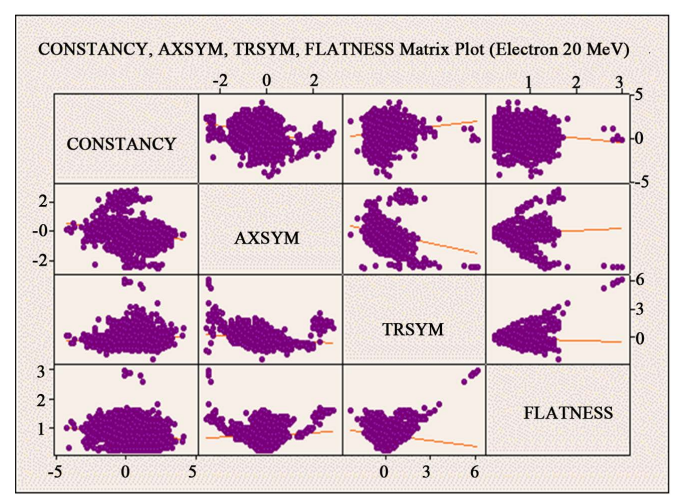

(g)

Figure 12. (a)-(g) Scatter-plot matrices of the four variables for all seven energies. 
results indicated that the response was generally consistent within each set of accelerators with different monitor chamber designs. The chambers used in their $2100 \mathrm{C} / \mathrm{D}$ Linac resulted in an increase in measured output over time for the first 4 years of use, which is nearly the same model as our Trilogy Linac. Similar to our findings, their output response trend appeared the same (upward shift) for all clinical energies used on the 2100C/D Linac. By tracking these changes it has been possible to predict the response over time to allow appropriate adjustments and gain confidence in machine performance. It has also provided data to indicate the need for planned preventative intervention and indicate if the monitor chamber response is behaving as expected. In our case, when visualizing our output and symmetry plots, we realized some unusual behavior (non-trending) starting from the end of 2014. By investigating further, we found out that the memory of the QABC+ was beginning to fail since that time. Then we sent out the unit to the manufacturer for repair including upgrading the firmware and software.

Bartolac and Letourneau [7] characterized underlying seasonal variations in Linac output over 3 years for 15 Linacs, in particular $6 \mathrm{MV}$ photon beams. Runtime plots of output revealed sinusoidal, seasonal variations that were consistent across all units, irrespective of manufacturer, model or age of machine. The average amplitude of the variation was on the order of $1 \%$. The peak and valley occurred in early April and September, respectively. Coincidentally our symmetric plots for all energies were observed almost the same pattern (period = 12 months; amplitude $=0.8 \%, 0.5 \%$ for photons, electrons, respectively). Characterization of cyclical seasonal trends allows for better separation of potentially innate accelerator behavior from other behaviors (e.g. linear trends), which could indicate service requirements.

Hossain [8] also reported that for 3 Linacs with 9 beams, output can increase by about 2\% - 4\% per year over a period of more than three years, if artificially removing the adjustments by physicists which were done once every 3 - 6 months. Similar to our setting, the output was measured using devices with ion chambers. Similar to our findings (Figures 6-10), they found quasi-periodic behavior manifested in the seasonally averaged output, showing annual variability with negative variations in the winter and positive in the summer. This trend was weakened when the daily output was normalized by the monthly-calibrated output, indicating that the variation of the periodic component may be intrinsic to both the Linacs and the daily measurement devices. To our understanding, the stability of some Linac components can vary with seasonal temperature changes, incoming power, and main circuit board, that affects the beam symmetry.

Uddin [9] studied short and long term drifts in outputs of Linacs by fitting empirical mathematical models to the QA measurements. They found that long-term drifts were well modeled $(\leq 1.5 \%)$ by either a straight line or a single-exponential function. A drift of $2 \%$ occurred in 12 - 18 months. The shortest drift times of only $2-3$ months were observed for some new accelerators just after the commissioning, but they stabilized during the first 2 - 3 years. The short-term reproducibility and the long-term stability of local constancy checks, carried out with a sealed plane parallel ion chamber, were also estimated by fitting empirical models to the QA measurements. The reproducibility was $0.3 \%-0.6 \%$ depending on the positioning practice of a device. Long-term instabilities of about $0.3 \%$ /month were observed for some checking devices. Luketina et al. [10] reported that one Varian Linac (SN42) was relatively stable with the output generally drifting between $+/-1 \%$ and the other newer linear (SN1027) had a consistent increase in the average output of about 2.5\% per year over a period of five years.

Overall, our output and symmetry trends are consistent with all reports in the literature for new Linacs using the sealed monitor chambers from the specific vendor. In this work, we used data visualization tools to efficiently evaluate the pattern of output fluctuation over a long period of time, and detect any unusual events in daily performance. Moreover, our analysis uncovers the correlation relationships between different variables, allowing the prediction of their behavior. This work shows a strong potential for applying data visualization tools to clinical medical physics field, in particular to improve the effectiveness of quality control program. Future works include developing new data visualization techniques, utilizing machine learning to predict the behaviors of variations in machine's output, and further exploring the correlation relationship among different features.

\section{Conclusion}

Data visualization is a new trend in the era of big data in radiation oncology research. It enables the past descriptive information not only be made more intuitive and enhanced support for data analyzing, but also can significantly enhance the expression of the pattern. Effective visualization helps physicists to predict the response over time and allows appropriate adjustments to maintain the Linac within tolerances. Data visualization is also an effective tool to perform data comparisons, alert failures, and potentially identify causalities. 


\section{Acknowledgements}

This research was funded in part through the NIH/NCI Cancer Center Support Grant P30 CA008748.

\section{References}

[1] Eric, E.K., et al. (2009) Task Group 142 Report: Quality Assurance of Medical Accelerators. Medical Physics, 36, 4197-4212. http://dx.doi.org/10.1118/1.3190392

[2] Kutcher, G.J., et al. (1994) Comprehensive QA for Radiation Oncology: Report of AAPM Radiation Therapy Committee Task Group 40. Medical Physics, 21, 581-618. http://dx.doi.org/10.1118/1.597316

[3] Smith, K., et al. (2015) AAPM Medical Physics Practice Guideline \#8: Minimum Practice Requirements for Linac QA. Journal of Applied Clinical Medical Physics. In Press.

[4] Polgar, I. (1999) Quality Control and Quality Assurance of Linear Accelerators. In: Mayles, W.P.M., Lake, R., McKenzie, A., Macaulay, E.M., Morgan, H.M., Jordan, T.J. and Powley, S.K., Eds., IPEM 81 Physics Aspects of Quality Control in Radiotherapy, The Institute of Physics and Engineering in Medicine, York.

[5] Chang, W. (2012) R Graphics Cookbook, Practical Recipes for Visualized Data. O’Reilly Media, Sebastopol.

[6] Grattan, M.W.D. and Hounsell, A.R. (2011) Analysis of Output Trends from Varian 2100/D and 600C/D Accelerators. Physics in Medicine and Biology, 56, N11-N19. http://dx.doi.org/10.1088/0031-9155/56/1/N02

[7] Bartolac, S. and Letourneau, D. (2015) Assessment of Seasonal Linear Accelerator Output Variations and Associated Impacts. Medical Physics, 42, 3363. http://dx.doi.org/10.1118/1.4924497

[8] Hossain, M. (2014) Output trends, Characteristics, and Measurements of Three Megavoltage Radiotherapy Linear Accelerators. Journal of Applied Clinical Medical Physics, 5, 1-15.

[9] Uddin, M.T. (2012) Quality Control of Modern Linear Accelerator: Dose Stability Long and Short-Term. Proceedings of IPAC2012, New Orleans.

[10] Luketina, I.A. and Greig, L. (2004) Linear Accelerator Output Variability. Australasian Physics \& Engineering Sciences in Medicine, 27, 155-159. http://dx.doi.org/10.1007/BF03178676 